Color Matching to Ink Jet Printers from a Computer Screen, Part 2.

\author{
Jerry Sedgewick \\ University of Minnesota \\ sedge001@umn.edu
}

In the previous article (part 1), the procedure for setting the computer screen was discussed by using Adobe Gamma in the control panel. Adobe Gamma is also found on the drive where the system is installed. Click on Program Files $>$ Common Files $>$ Adobe $>$ Calibration $>$ Adobe Gamma.cpl. Double click to launch the gamma control wizard. On a Macintosh the Apple Calibration Utility can be used.

The merits of using ink jet printers were also discussed. Merits include low cost (though costs escalate with the purchase of cartridges), high quality prints from any application, and the technology of a fine spray with higher image resolution as opposed to a laser jet printer and Tektronix Phasers (these printers separate images into dots at compromised resolutions). Certainly any printer other than a laser jet can be used for color matching at satisfactory resolutions when budgetary considerations are not important, such as higher end photographic printers (e.g., the Fuji Pictrography printer) or thermal printers (e.g., those made by Codonics and Kodak).

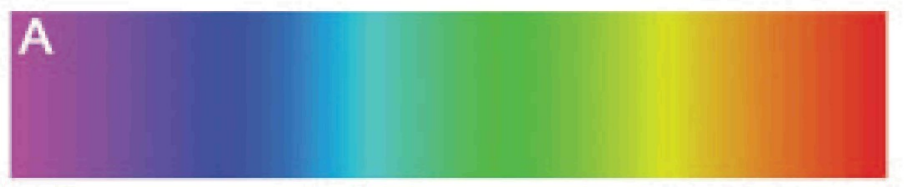

B

Fig 1. A) Top image shows the visible spectrum after being converted from the primaries of light (Red, Green, Blue or RGB) to the primaries of pigments (Cyan, Magenta, Yellow and the addition of Black or CMYK). This was done primarily in the Hue \& Saturation dialogue box by de-saturating with the Saturation slider (all colors print if de-saturated to varying degrees). B) Bottom image shows ranges of colors that do not translate well from RGB color to CMYK. These are covered in neutral gray, when using Gamut Warning in Photoshop. The RGB image was slightly de-saturated to emphasize colors more likely to convert from RGB to CMYK without loss of highlights and shadows (cyan, yellow and much of red). Note the wide range of gray covered hues that present trouble when printed.

Printers, however, which are lower end, such as thermal printers that print from a color video monitor, do not have the range of colors and gamma options for satisfactory prints. Printers available at chain stores as self-serve stations can be more trouble than they're worth, for these may not be calibrated with changes in paper stock and with heavy use over time. Neither of these printing options are recommended when striving to match monitor colors with prints.

A dedicated computer and ink jet printer for color balancing create a work area in which results are repeatable, predictable and which allow for comparisons to various outputs. Don't expect to match colors and contrast, however, from PowerPoint or Word. These programs aren't intended for color matching, and neither display red, green and blue numeric values, making color matching problamatic.

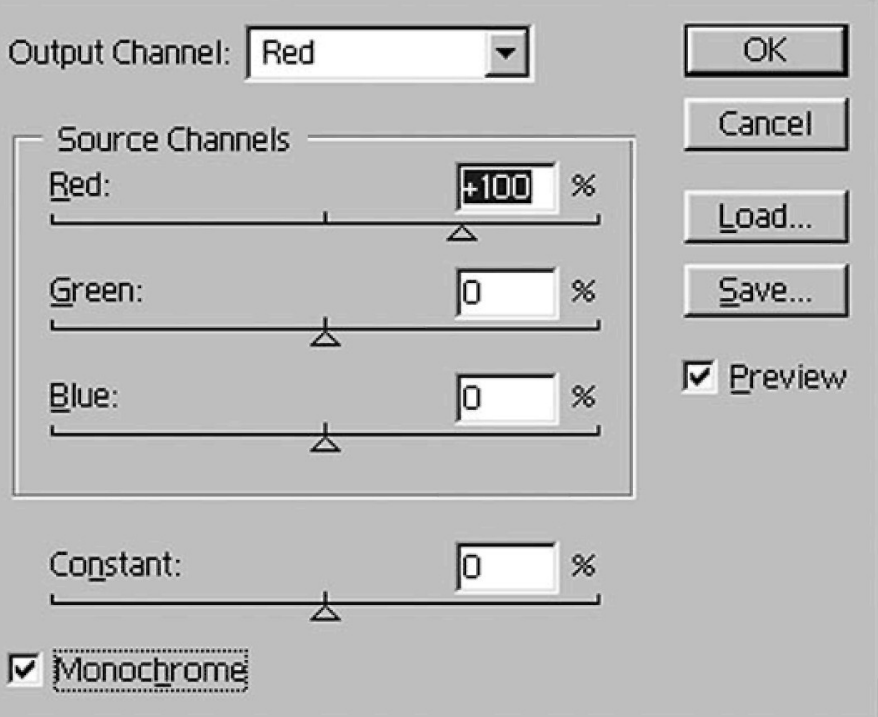

Fig 2. Channel Mixer should be used to convert from color to grayscale when one color predominates. These include darkfield images, such as those of fluorescing objects. Choose the predominant color for Output Chanel, then check Monochromatic (Output Channel will display gray). This protocol preserves luminance values when converting from color to grayscale.

The trouble with Scientific Images. The reason that colors generated in research so often do not match the print result from two phenomena: First, the primary colors for light (red, green and blue) comprise a range of colors (known as gamut) simply not duplicated in the range of colors made from primary colors for pigments (cyan, magenta, and yellow: referred to as CMYK with the $\mathrm{K}$ representing black). Because of that, the hues that go from blue to red-blue (violet) become dark when printed, but green-blue (cyan) remains as bright as it appears on the computer screen. The out-of-gamut RGB colors also include shades of green, red and orange (see fig 1).

Secondly, colors are often saturated (intense colors farthest away from admixture with gray). These are represented by the unmixed colors in the visible spectrum: violet, blue, green, orange, yellow and red. More saturated colors of certain hues exist at the outer edges of the CMYK gamut than less saturated colors, making these colors that much more difficult to reproduce on paper. Not many of these saturated colors exist in our natural environment-the one that we photograph when on vacation-and that is why we do not generally experience this problem outside the realm of scientific images. We are lead to believe by past experience with standard photography that color matching and reproduction shouldn't be so difficult. Add to that those of us who made $35 \mathrm{~mm}$ slides for slide presentations. We worked for a period of time with PowerPoint and its very reproducible deep blue colors on film. But this process was one of RGB color backlit by a computer screen and the slides were then backlit just like the computer screen. That process hardly compares to a complete conversion of all RGB colors at each pixel to the non-overlapping gamut of CMYK on a paper surface that reflects color from ambient light.

Color Matching with Saturated Colors: Prints are more likely to match a screen when using RGB colors closer to the gamut of pigments. Thus, it is important to choose RGB color values carefully, 


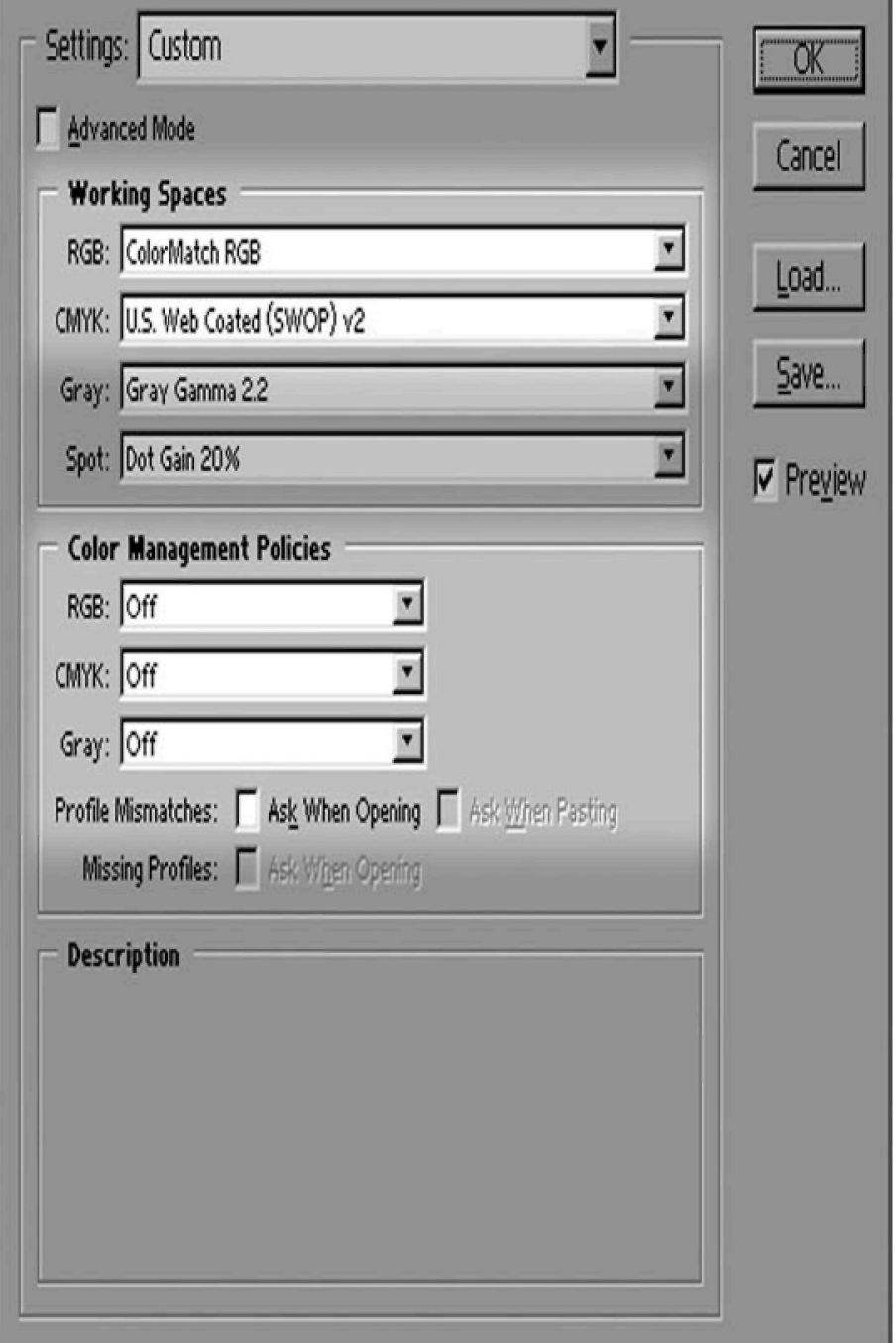

Fig 3. The color settings indicated in this figure are good choices when matching the computer screen to what is printed on paper. If the printing occurs on a press used in Europe or Japan, choose these settings from the drop down list instead of US Web Coated (SWOP) v2. To avoid prompts when opening images, you may wish to uncheck color management and color profile mismatch warnings.

especially when using color ranges that are saturated. Then, if color still does not match, printer values can be changed to fine tune the color. This print, then, becomes the proof sent to a publisher along with the digital image. Or it becomes the kind of image with colors that can be easily tweaked when creating a poster, or placing images into grant applications, reports, brochures, and other paper-based hard copy. As an added note, make sure you use high quality paper meant for ink jet printing. In our lab, we have found Cougar paper to be ideal for standard paper. For glossy photographic paper, we look for a high white opacity.

This article will be limited to only the saturated colors used with fluorescently labeled samples, the reds, greens and blues that are difficult to convert to CMYK. These include the popular red, green and blue colors used as representations of fluorophores; along with violet and orange used in psuedocoloring. Yellow, cyan, magenta, and many of the red shades overlap with RGB values, and so these will not be covered in this article.
Start with the Grayscale Image. Often RGB or Indexed Color images have been colorized either in software or via hardware algorithms by manufacturers of confocal systems and CCD cameras. Attempting to change a pre-colorized image can lead to a great deal of frustration, because $100 \%$ of the chosen color is used without an admixture of any other color. Because CMYK requires the addition of other colors, the only means for tweaking values to fit the CMYK gamut lie in replacing colors, especially when more than one color exist (in Photoshop, under Image $>$ Adjustments $>$ Replace Color); or in adding colors to the existing image (in Photoshop, under Image $>$ Adjustments $>$ Variations). These protocols can be used when original grayscale images cannot be located. The explanation for how to use these methods, however, lies outside the scope of this article.

Making Grayscale from RGB Color or Indexed Color images. If your images are colorized for one color to represent, say, one fluorophore, then this kind of image can easily be made into grayscale. First you may want to check your colorized image to see if values have been made with the idea that these might end up as hard copy on paper versus pretty pictures on a computer screen, something you'll learn how to do as you read on (use gamut warning). Given that this is an unlikely scenario, proceed by unmixing the red, green and blue channels. Do NOT simply convert the image to grayscale or luminance values will be lost. That's because "grayscale" is obtained from a color image by using only the green channel, the same channel, in effect, most strongly used by the human eye.

If the image is an Indexed Color image, then change its mode to RGB Color first. Do so under Image $>$ Mode $>$ RGB Color. If the image is 16 bit, first type in values of $12-16$ (depending on saturation of whites in the image) in the Input Levels box on the right in the Levels dialogue box (Image > Adjustments > Levels), or use a filter described in an earlier article in Microscopy Today. Then convert the image to 8 bit (Image $>$ Mode $>8$ Bits/Channel).

To unmix the channels, under Image $>$ Adjustments, use Channel Mixer. In the Channel Mixer dialogue box, choose the prominent hue next to Output Channel, and check Monochrome at the bottom left (fig 2). This method will preserve the inherent

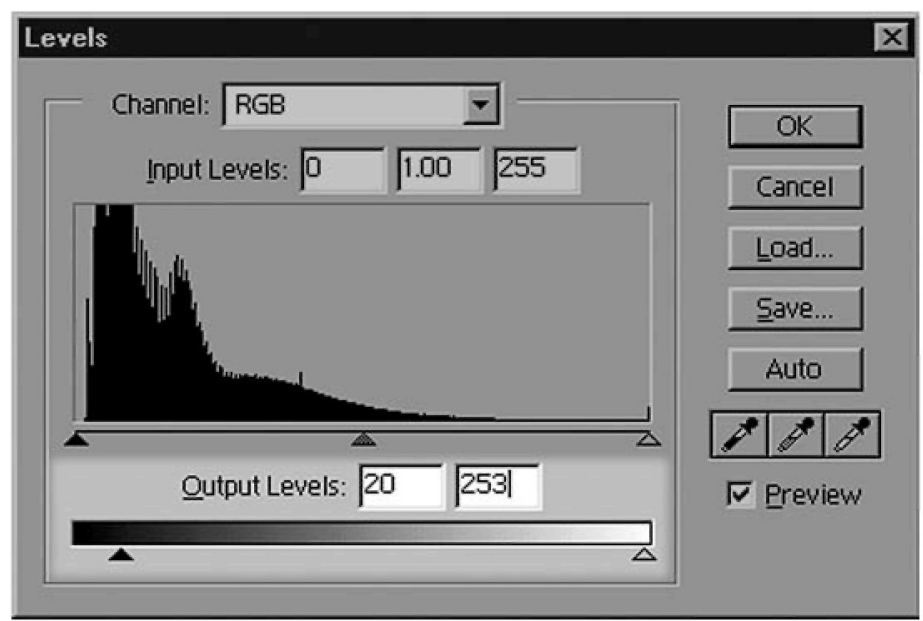

Fig 4. The Levels dialogue box shows Output Levels at the bottom. The box on the left decreases the intensity of black; on the right the intensity of white. These can also be set in each color channel to yield a colorized image from a grayscale image. Suggested numeric values are shown in Fig 5. 


\begin{tabular}{l|l|l|l|l}
$\begin{array}{l}\text { COLOR } \\
\text { DESIRED }\end{array}$ & $\begin{array}{l}\text { RED } \\
\text { OUTPUT } \\
\text { VALUE }\end{array}$ & $\begin{array}{l}\text { GREEN } \\
\text { OUTPUT } \\
\text { VALUE }\end{array}$ & $\begin{array}{l}\text { BLUE } \\
\text { OUTPUT } \\
\text { VALUE }\end{array}$ & $\begin{array}{l}\text { SHADE } \\
\text { OF } \\
\text { COLOR }\end{array}$ \\
\hline GREEN & 110 & 0 & 160 & $\begin{array}{l}\text { Bluer } \\
\text { Green }\end{array}$ \\
\hline GREEN & 160 & 0 & 140 & $\begin{array}{l}\text { Yellower } \\
\text { Green }\end{array}$ \\
\hline RED & 0 & 40 & 50 & Red \\
\hline RED & 0 & 120 & 50 & $\begin{array}{l}\text { Oranger } \\
\text { Red }\end{array}$ \\
\hline BLUE & 60 & 160 & 0 & $\begin{array}{l}\text { Cyan } \\
\text { Blue }\end{array}$
\end{tabular}


Fig 5. The table at the top indicates suggested numeric values for making colorzed images from a grayscale image. These values may change depending upon the nature of the image, but indicated values serve as good starting points. Image $A$ uses the yellowish green values indicated in the table, image $B$ shows the image when $100 \%$ green is used with no other colors (much the same as many colorized images from manufacturers) and image $C$ shows a green colorized image shifted more to blue. Carefully note the lack of tonal range in Image $B$ when compared to $A$ and C. Likewise, Image $D$ shows a yellow shifted red colorized image; E shows a $100 \%$ red image without green or blue; and $F$ shows a blue shifted red image. The differences in the reds are subtle, but the addition of green and red values when colorizing yields images with greater tonal range and contrast. Image $G$ shows $100 \%$ blue without additional red and green colors. This color of blue prints close to black. By shifting the blue toward cyan, as in image $H$, the color brightens. Steps to colorize images can be automated by using actions. Check the quickphotoshop.com website for actions to download.

contrast in the image.

Colorizing or Re-Colorizing the image: Color Settings (see fig 3). Before any colorizing takes place, Color Settings need to be set in a manner best suited for scientific images for several possible outputs. Photoshop attempts to fine tune CMYK color based upon the printing press or other output for the image. For example, on European presses the gamut of CMYK color is different than in the U.S. Also, more ink can be piled on coated (shiny, magazine paper) versus uncoated paper (typewriter paper, newspaper, non-shiny and absorbent paper), affecting the values in the shadow regions. And web presses like that used to print this magazine run at such high speeds that not as much ink is used as in sheetfed presses. The output can be chosen in Photoshop, as a way of evaluating images reliably in the gamut of your final output.

Choose the CMYK color settings in Photoshop 6 and greater under Edit on the menu. Select Color Settings, and then, in the CMYK list item choose the correct selection. If that is unknown, a good default setting is US Web Coated. (SWOP) 22.

The input RGB color space also needs to be chosen. The default is $s R G B$, but the better setting for color matching to paper output is Color Match RGB. This setting displays and emulates red, green and blue values on the basis of output to printed paper.

While here, you can uncheck Profile Mismatch and turn off Color Management Policies to avoid annoying prompts when opening images in Photoshop. These are not essential tools for scientists, rather these are useful tools for those involved in printing presses and publication.

Limiting Range of Whites and Blacks (see fig 4). Color has been the subject matter thus far in speaking to the limitations of printing, but deep blacks may lose details and the whitest whites may not print at all, or these areas can resist being colorized. Furthermore, lightening the deepest blacks can aid in conversion to printable values.

To limit the grayscale range, especially in the blacks, under Image $>$ Adjust, select Levels. This dialogue box displays a histogram showing the number of pixels on the $y$ axis and grayscale values that lie at and between 0 and 255 on the $\mathrm{x}$ axis. The Input Level box values increase the white and black values, as well as the black and white triangle sliders. The Output Level box values decrease white and black values, as well as the triangle sliders under its gradient. Limit the range, then, by typing in values in the Output Level box. Generally, no difference in deepness of the darkest black exists between 0 and 20 on the black output levels, so a value close to 20 should be entered in the black (left) output level box (the best value can be tested iteratively on your printer). To be sure that the oversaturated whites (untextured whites at 255) can print and be colorized, set the white output level box to 252 or 253 . Generally, this level of white differentiates the whites in the image from a white border because it is slightly off-white.

At this point, the gamma slider (the middle slider under the histogram) can be used to increase or decrease contrast in the midtones. Midtone values reproduce faithfully on printers and printing presses.

Change the Grayscale image to $R G B$. Before colorizing, the grayscale image needs to be changed to RGB Color. Under Adjust $>$ Mode, select RGB Color.

Activating Gamut Warning. Before colorizing, under View, 
select Gamut Warning. This will cause gray to shade areas where RGB values do not fit within the CMYK gamut. When a flat gray color covers parts of an image, as in figure $1 \mathrm{~B}$, this is an indication that the covered colors are "out of gamut,"and will print dull and flat. A few patches of gray, however, will not create printing problems. It's a good idea to memorize the keystrokes for turning on and off Gamut Warning, as these can be used when a dialogue box is open. The keyboard strokes are Control/command $+y$.

Colorizing the Image. The best method for colorizing an image can be accomplished by using the Levels or Curves dialogue boxes. In both methods, the output level is decreased in the color channels NOT desired. For example, if green is desired, the output level is decreased in the undesired red and blue channels, leaving the color green.

Using Levels. Under Image > Adjust, select Levels. To select a channel in order to decrease the output levels, click on the arrowhead next to the Channels box and select the appropriate channel from the drop down list. Decrease the output levels by entering numeric values in the output box on the right, or by using the white slider and moving it to the left. As the Output Level values decrease, the likelihood of activating the gamut warning gray overlay to indicate unprintable colors increases. Use the table (fig. 5) to set red, green and blue values to create desired and printable fluorescent colors

These colors can be changed to CMYK mode and further tweaked using Levels to adjust contrast (input levels) or by using Hue \& Saturation to increase or decrease color saturation (using the saturation slider), or to shift overall color slightly (using the Hue slider). Once changed to CMYK mode, all changes to color will be printable, and a match between the printer and the screen will be far better than previous experience.

The CMYK image can be saved, and then returned to $R G B$ Color for future use, depending on the output (save the RGB image as well). If the output is the printing press, then the CMYK image is sometimes preferred, but be sure to send a proof. If the destination for the image is a poster printer, ink jet printer, inclusion in Word or PowerPoint, then the RGB image is often necessary.

More complicated multi-colored images and ways to tweak images to make prints that match a computer screen will be discussed in the next article in Microscopy Today.



\section{Nanoprobe Dreams?}

For images cleaner than new fallen snow, Here is what Microscopists should know, To improve resolution And reduce contamination Rely on Evactron ${ }^{\circledR}$ plasma glow.

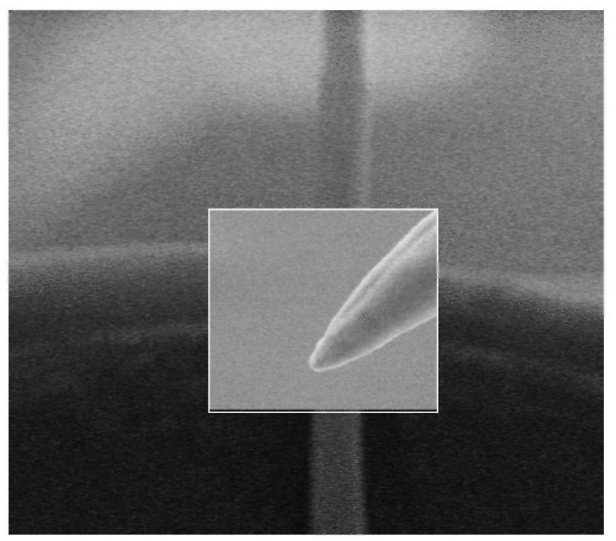

The Zyvex ${ }^{\circledR}$ Nanoprobe stays clean after 30 minutes of scanning because of Evactron ${ }^{\circledR}$ cleaning.

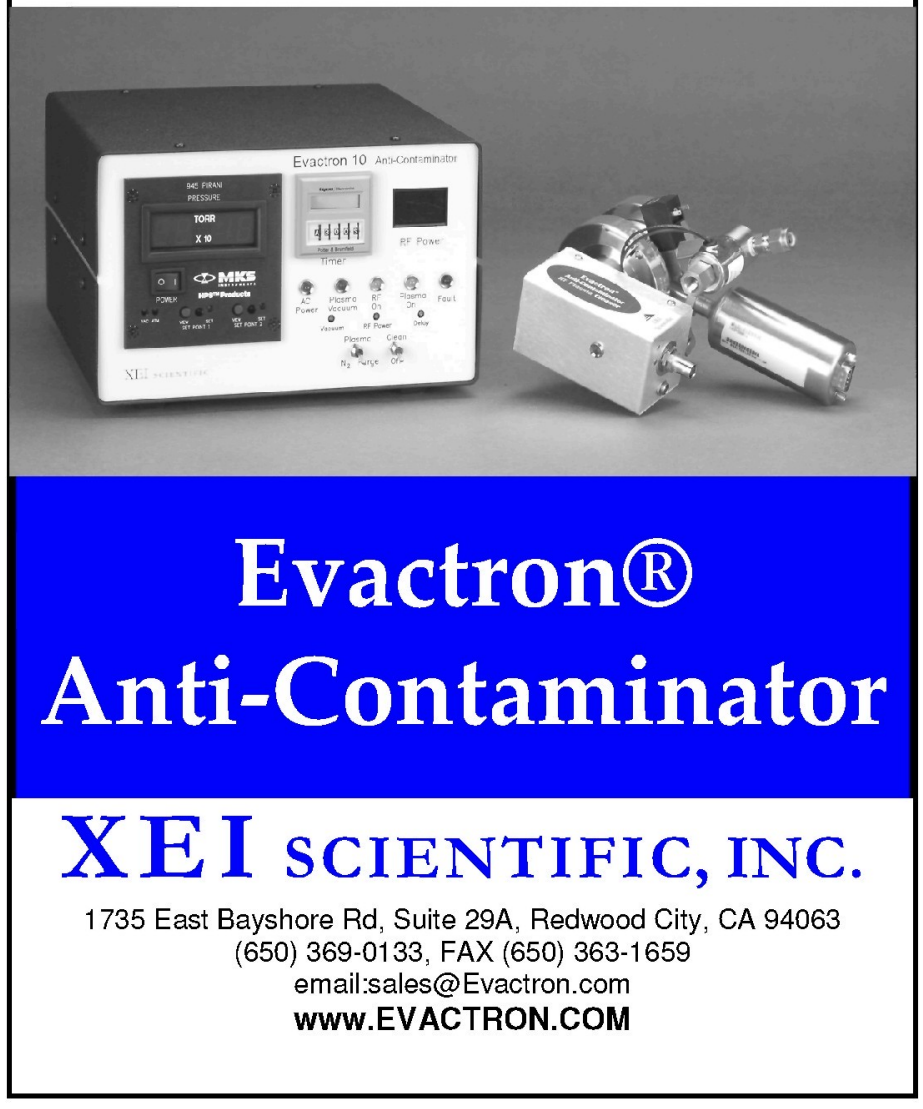

